\title{
Comprehensive supervised heavy training program versus home training regimen in patients with subacromial impingement syndrome: a randomized trial
}

\author{
Pierre Schydlowsky ${ }^{1 *}$ (D, Marcin Szkudlarek ${ }^{1,2,3}$ and Ole Rintek Madsen ${ }^{4}$
}

\begin{abstract}
Background: There is no consensus on the best training regimen for subacromial impingement syndrome (SIS). Several have been suggested, but never tested.

The purpose of the study is to compare a comprehensive supervised training regimen (STR) based on latest evidence including heavy slow resistance training with a validated home-based regimen (HTR). We hypothesized that the STR would be superior to the HTR.

Methods: Randomised control trial with blinded assessor. 126 consecutive patients with SIS were recruited and equally randomised to 12 weeks of either supervised training regimen (STR), or home-based training regimen (HTR). Primary outcomes were Constant Score (CS) and Shoulder Rating Questionnaire (SRQ) from baseline and 6 months after completed training. Results were analyzed according to intention-to treat principles. The study was retrospectively registered in ClinicalTrials.gov. Date of registration: 07/06/2021. Identification number: NCT04915430.

Results: CS improved by 22.7 points for the STR group and by 23,7 points for the HTR ( $p=0.0001)$. The SRQ improved by 17.7 and 18.1 points for the STR and the HTR groups respectively $(p=0.0001)$. The inter-group changes were non-significant. All secondary outcomes (passive and active range of motion, pain on impingement test, and resisted muscle tests) improved in both groups, without significant inter-group difference.
\end{abstract}

Conclusion: We found no significant difference between a comprehensive supervised training regimen including heavy training principles, and a home-based training program in patients with SIS.

Keywords: Shoulder, Rotator cuff, Subacromial impingement syndrome, Training, Heavy slow resistance training

\section{Background}

Subacromial impingement syndrome (SIS) is the most common cause of shoulder pain. According to the definition adopted, it explains 30 to $86 \%$ of all cases with shoulder pain $[1,2]$. The condition affects the rotator cuff tendons, especially the supraspinatus tendon [3]. Exercise

\footnotetext{
*Correspondence: pierre.schydlowsky@dadlnet.dk

${ }^{1}$ Reumatologiklinikken i Værløse, Bymidten 11,1, 3500 Værløse, Denmark

Full list of author information is available at the end of the article
}

therapy has been shown to reduce pain and improve function [4-8].

Even though most training regimen include strengthening exercises to the rotatorcuff and the scapula, there is still no consensus on which training regimen to recommend, neither regarding the type, number, and intensity of exercises, nor their duration and frequency. In a newly systematic review and metaanalysis, supervised training and self training resulted in equal improvement of pain and function, and larger improvement than no original author(s) and the source, provide a link to the Creative Commons licence, and indicate if changes were made. The images or other third party material in this article are included in the article's Creative Commons licence, unless indicated otherwise in a credit line to the material. If material is not included in the article's Creative Commons licence and your intended use is not permitted by statutory regulation or exceeds the permitted use, you will need to obtain permission directly from the copyright holder. To view a copy of this licence, visit http://creativecommons.org/licenses/by/4.0/. The Creative Commons Public Domain Dedication waiver (http://creativeco mmons.org/publicdomain/zero/1.0/) applies to the data made available in this article, unless otherwise stated in a credit line to the data. 
training for patients with subacromial pain syndrome. The included studies used various exercises and dosages, but all included elements of rotatorcuff and scapular muscles strengthening [6].

In another systematic review and meta-analysis of patients with SIS, eccentric exercises provided no improvement of function compared with other exercises. The exercise regimens showed both similarities and differences, emphasizing the disparity of conceptions as regards to training. All interventions focused on at least one of these two exercises: shoulder external rotation with the shoulder in neutral position, and shoulder abduction in the scapular plane with the thumb pointing up. There was no consensus on the duration of training, which ranged from 4 till 12 weeks, nor on the frequency or training, which varied between 2 times per week to 2 times per day. Painful exercises showed no significant differences compared to pain-free exercises, and training regimens of $6-8$ weeks were almost as effective as 12 weeks training [8].

Based on a systematic review, Kuhn [9] has suggested an exercise regimen including a combination of motion, stretching, and strengthening of the rotator cuff and of the scapular muscles. This regimen has not been tested, but several studies support this approach [10-19]. Yet, to this day, there is no consensus on which training regimen to recommend.

Training programs based on strengthening eccentric exercises for the rotator cuff and strengthening concentric/eccentric exercises for the scapula stabilizers may prevent surgery and yield year-long lasting effects $[10$, 11]. Recent studies have emphasized the importance of including infraspinatus, trapezius inferior and serratus anterior muscles in the rehabilitation of SIS [10, 12-14].

Stretching of the shoulder can improve range of motion and function, and prevent muscle strain $[15,16]$, and stretching of the pectoralis minor and of the posterior capsule as well as proprioceptive training are often suggested [13, 14, 17], even though the effects of pectoralis minor stretching only have been documented in scapular kinematics outcomes. There may even be doubts about it's clinical efficiency, as Gutiérrez-Espinoza et al. found no short term benefit of additional pectoralis minor stretching on a specific exercise program, in terms of functional improvement or pain reduction in patients with subacromial pain syndrome [20]. Those findings have to be confirmed though.

Correction of posterior shoulder tightness has been documented in patients with internal impingement syndrome $[13,16]$. Even though it isn't as well documented in SIS, it makes sense to restore flexibility deficits, as they may lead to scapular malpositioning [13]. Besides, two recent studies suggest that posterior shoulder stretching may improve pain and function in patients with SIS [18, 19].

Lately, there has been focus on tendon training, and heavy slow resistance training (HSRT) has reduced pain, and yielded high patient satisfaction for the rehabilitation of Achilles and patellar tendinopathy [21]. Besides, only heavy load training compared to moderate training seems to maintain tendon mechanical properties in old age [22]. We therefore decided to incorporate heavy training as well, even though it had not been tested on SIS, finding it likely that rotatorcuff tendons would react positively, tendons in the upper extremity having same mechanic and physiologic properties as the ones in the lower extremity.

In this study, we hypothesized, that a supervised exercise protocol (STR) based on motion, stretching, and muscle and tendon strengthening with HSRT and focus on both scapula stabilizing muscles, and rotator cuff tendons, would be superior to a simpler home exercise program that resulted in higher function score, and shoulder satisfaction than the untreated control group [5]. For the STR, exercises that most effectively stimulate scapular and rotator cuff muscles according to electromyography studies and a systematic review were chosen $[9,23]$. The purpose of the study was not to examine the effect of a single parameter but to compare two different training regimens as a whole.

\section{Methods}

In a randomised control trial, 188 successive patients referred to our clinic by their general practitioner from September 2013 to November 2017 were considered for participation. 126 patients fulfilled the inclusion criteria and agreed to participate. A CONSORT flow diagram is shown in Fig. 1. Allocation of the patients to either a supervised training regimen (STR) or a home training regimen (HTR) was concealed. All data were collected at our clinic.

Oral and written consent was obtained from all patients. Even though duration of symptoms wasn't recorded, patients waited on average 4 months between time of referral from the General Practitioner and time of consultation in our clinic, due to the structure of the Danish system. There were thus no patients with duration of symptoms less than 4 months.

The 2 groups differed widely in terms of number of exercises and duration of training, the STR group having far more exercises and being more time-consuming than the HTR, the purpose of our study being to find out whether there was any benefit of the much more comprehensive STR program compared to the simpler HTR as a whole. 


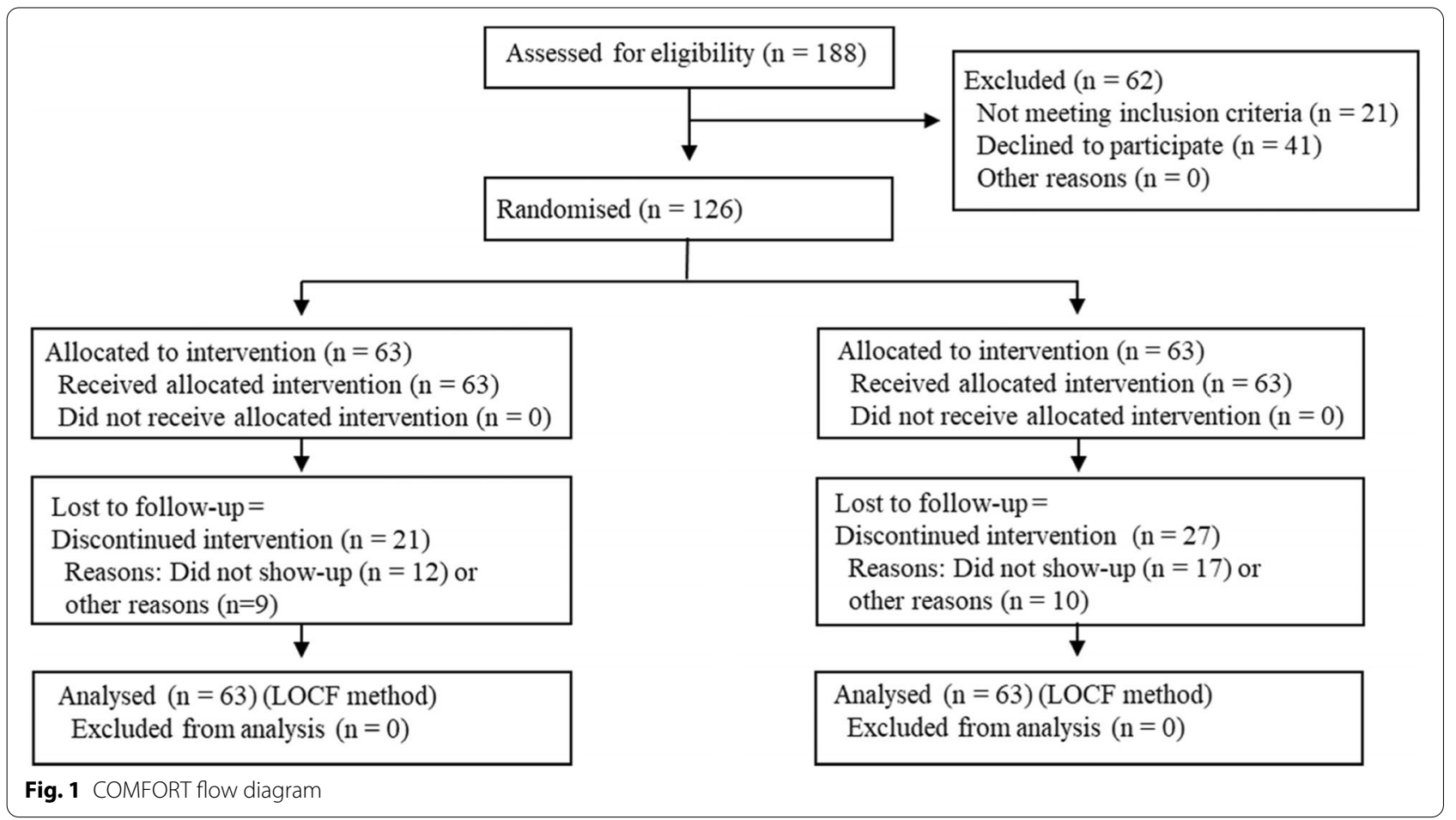

Training of participants in the STR group was supervised one-on-one by the same physiotherapist. Participants in the HTR group were provided with written instructions and shown how to perform exercises by the physiotherapist. They were assessed a week later to make sure that the exercises were performed correctly. No participant received any steroid injection during the trial.

The study was approved by the Ethics Committee of the Capital Region of Denmark on april 24th. 2013, protocol nr. H-4-2013-030.

\section{Inclusion criteria}

- Subacromial impingement syndrome, regardless of age, sex, employment status, activity level, cause (trauma or overuse), and the severity and duration of symptoms. In order to fulfil inclusion patients had to reveal 3 positive tests out of 5: Positive Neer's test, positive Hawkins test, positive Jobe's test, painful arc, and pain on resisted external rotation of the arm [24]. All participants were examined with an ultrasound scanner at inclusion.

\section{Exclusion criteria}

- Ongoing claim with the Labour market insurance, insurance company or comparable institution.
- Ongoing application for job revalidation or health related pension.

- Radiating neck pain.

- Ultrasound verified complete rotator cuff tendon lesion, as defined by hypoechoic or anechoic full thickness defect of the tendon, or absence of the tendon.

- Periarthritis humeroscapularis.

- Acute luxation or fracture of the shoulder.

- Ongoing steady analgetic treatment of other concomitant painful condition, unrelated to the patients shoulder problem.

Flow

- All patients that fulfilled inclusion criteria, and did not have any exclusion criteria were offered participation in the trial. Age, sex, and self-reported employment status were recorded.

- All patients that accepted participation, were randomised blindly to either a supervised training regimen (STR) or a home training regimen (HTR).

- Participants in the STR group were supervised in the clinic.

- Participants in the HTR group were instructed in a home-based exercise regimen. 
- All participants were examined with an ultrasound scanner at inclusion.

\section{Randomisation procedure}

An equal amount of cards marked either group I (STR) or II (HTR) were folded and mixed, as to conceal the group for the examiner and the patient, who then picked up a card randomly.

\section{Training regimens}

\section{Supervised heavy training regimen (STR) [4, 9]}

This training regimen consisted of 3 phases: the 1st focused on restoring motion, the 2nd on strengthening of the rotator cuff muscles, and the 3 rd on strengthening of the scapular muscles. Stretching completed every training session.

- Motion training consisted of 6 exercises including postural and glenohumeral training.

- Postural training consisted of shoulder shrugs and shoulder retraction exercises.

- Glenohumeral training consisted of pendulum exercises, and active assisted flexion, abduction and external rotation. Motion training ended when the full passive flexion, abduction and external rotation had been re-established.

- Strengthening of the rotator cuff included 3 exercises: side lying external rotation, internal rotation and scaption.

- Strengthening of the scapular muscles included 5 exercises: press-up, push-up with a plus, serratus anterior supine punch, standing rowing (low row), and seated rowing (high row).

- Training sessions ended with 4 stretches: anterior shoulder stretch, posterior shoulder stretch, inferior capsular stretch, sleepers stretch.

Exercises were performed 3 times a week, with progression after the 1st week, and thereafter every 2 nd week.

\section{Choice of exercises}

\section{Motion exercises:}

Our choice of motion exercises was based upon a systematic review, where Kuhn suggested that initial rehabilitation should include postural training with shoulder shrugs and shoulder retraction, pendulum exercises, and active assisted motion which have all been described and used in other studies [9, 25, 26]. Codman's pendulum exercises are commonly prescribed after shoulder surgery and injury to provide distraction and oscillation resulting in decreased pain, increased flow of nutrients into the joint space, and early joint mobilization [27]. Some studies have questioned their relevance in SIS rehabilitation. Thus in a study that aimed at determining if added weight affected the shoulders ability to relax, Ellsworth et al. [27] showed that generally, the supraspinatus/upper trapezius muscle activity was significantly higher than the deltoid and infraspinatus activity - especially in the patients with pathological shoulders. The pendulum exercise with or without weight, did not have a significant effect on shoulder EMG activity though, neither in normal nor in pathological shoulders. And in another study on subjects having undergone subacromiel decompression [28], EMG analysis of the rotatorcuff showed that the supraspinatus tendon remained as passive during pendulum exercises as at resting baseline. It is therefore safe to assume that these exercises presented no risk in our population, but they may not be efficient for restoring passive shoulder range of movement, as a recent study on shoulder kinematics [29] has demonstrated that Codman pendulum exercises depend mainly on truncal movement and produce very little movement in the Glenohumeral and Scapulothoracic joints.

\section{Strengthening exercises:}

We combined a series of exercises, which value have been emphasized by several authors $[5,12,13,17,23$, 30-35].

\section{- Side lying external rotation}

The importance of including the external rotators in rehabilitation programs has been emphasized by several studies [12, 13, 30, 31].

Experimental shoulder pain elicited by injection of hypertonic saline in the supraspinatus muscle has an inhibitory effect on the activity of the infraspinatus muscle during arm elevation, but not on the activity of the scapulothoracic muscles. This indicate that it may be of importance to include strengthening of the infraspinatus in rotator cuff rehabilitation protocols [30]. Chaconas et al. [31] used a program basically consisting of standing resisted scapular retraction and posterior capsular stretch. Adding resisted external rotation was superior to active unresisted shoulder motion. We chose sidelying external rotation, as this exercise has a low Upper trapezius (UT)/Lower trapezius (LT) ratio, which is beneficial due to excessive UT activity and deficient LT activity in SIS $[12,13]$. 
- Internal rotation

We included internal rotation training in order to ensure balance between internal and external rotators [32], as it has been speculated that weakness of the internal rotators may lead to shoulder pain [33].

- Scaption

Scaption stimulates the UT, but also the LT, the middle and lower serratus anterior (SA), the rhomboids, the levator scapulae and the supraspinatus [23]. The exercise probably offers the opportunity of training the supraspinatus with HSRT, and with the exception of the UT, stimulates the function of the scapular muscles, that is essential for shoulder recovery $[12,13,17]$. Besides, it makes sense to target the supraspinatus in rehabilitation programs, as it is the most frequently affected rotator cuff tendon [3]. In a small study on 11 patients waiting for subacromial decompression surgery, eccentric training of the supraspinatus muscle, resulted in 2 out of 4 patients cancelling their planned operation [36] • Press-up

This exercise puts focus on the pectoralis major muscle and the latissimus dorsi. These muscles generate power. Jobe and Pink suggested that they ought to be included as part of a comprehensive rehabilitation program, based upon stimulation of the shoulders protectors, positioners, pivoters, and propellers, which they named the 4P [34].

- Push-up with a plus

Push-up with a plus effectively stimulates the middle and lower serratus anterior, and the pectoralis minor muscle [23].

\section{- Serratus anterior supine punch}

The exercise has emphasis on the serratus anterior, whose decrease in activity has been linked to alterations in scapular and humeral motion during arm elevation [5].

\section{- Standing rowing (low row)}

Low row has been recommended as an exercise to stimulate scapular muscles without putting strain on the rotatorcuff [35]. Rowing effectively stimulates UT, MT, LT, rhomboids, and the levator scapulae [23].
- Sitted rowing (high row)

We included high rows to stimulate the upper part of the scapular stabilizing muscles in order to provide participants with a comprehensive rehabilitation program.

\section{- Stretching exercises}

Tightness of the pectoralis minor muscle, the posterior capsule and/or external rotators may lead to scapular dysfunction [37]. The unilateral pectoralis minor stretch is an efficient stretching method for this muscle [38] and may lead to less shoulder pain and improvement of function [39]. Stretching of the posterior shoulder structures with sleepers stretch and cross-body stretch can improve range of motion (ROM) and function $[15,16]$. We added stretching of the inferior capsule to restore normal shoulder motion as well.

\section{Home based exercise (HTR) [5]}

This program has been validated [5]. The program consisted of the following:

- 1 motion exercise: upper trapezius relaxation

- 3 strengthening exercises: serratus anterior strengthening exercise, humeral external rotation with the arm at the side of the body, and humeral external rotation with a rubber band and the arm at 90 degrees abduction.

- 2 stretching exercises: posterior shoulder and pectoralis minor stretching,

Exercises were performed daily, with weekly progression.

Both the STR and the HTR groups exercised for 3 months and were encouraged to continue exercising on their own afterwards in case of persisting symptoms.

\section{Detailed training regimens}

Supervised training regimen (STR)

- Motion training

- Postural training

- shoulder shrugs

- shoulder retraction

- Glenohumeral training

- Pendulum exercises

- Active assisted motion (with the help of the opposite arm, cane, or training cable)

- flexion

- abduction

- external rotation 
Ad shoulder shrug and shoulder retraction: position is held for $10 \mathrm{~s}$. Then pause for $10 \mathrm{~s}$. Is repeated $\times 5$.

Ad pendulum exercises: 20 small circular movements clockwise, 20 small circular movements anticlockwise, 20 small movements in the sagittal plane, and 20 small movements in the frontal plane.

Ad active assisted motion: the arm is slowly moved to the desired position and back again. Is repeated $x 5$.

Motion training ends when the full passive flexion, abduction and external rotation has been re-established.

- Strengthening of the rotatorcuff

- Side lying external rotation

The patient is lying on the opposite side. External rotation is performed with a hand weight or a rubber band

\section{- Internal rotation}

The patient is sitting or standing. Internal rotation is performed with a hand weight or a rubber band.

\section{- Scaption}

The patient is sitting or standing. The elbow is held in full extension, as the arm is lifted in the scapular plan, till 90 degrees elevation. Resistance is provided by hand weight or a rubber band.

- Strengthening of the scapular muscles

- Press-up

The patient is sitting on a chair, and grabs the side of the chair with each arm. The patient then lift the upper body from the chair.

\section{- Push-up with a plus}

The patient is lying prone on the floor. Push-ups are performed "with a plus" with protraction of the scapulas and hyper kyphosis of the thoracic spine, according to the description of Jobe et al. (26)

\section{- Serratus anterior supine punch}

The patient is lying supine on the floor or on a couch. A hand weight is held in each hand, and the arms are flexed 90 degrees, with the hand weights pointing toward the ceiling. The patient then lifts both shoulder blades up toward the ceiling.
- Standing rowing (low row)

Low rows are performed with the patient standing or sitting with the arms at 0 degree of flexion.

- Sitted rowing (high row)

The patient is sitting with the arms flexed 90 degrees, allowing high rows to be performed.

- Stretching

- Anterior shoulder stretch

The exercise is performed with the arm lifted up to 120 degrees of abduction, or lower if this position illicits pain.

- Posterior shoulder stretch

The arm is lifted up to 90 degrees, or lower if this position illicits pain. The opposite hand grabs the backside of the elbow and presses it towards the opposite shoulder.

- Inferior capsular stretch

The arm is lifted in full abduction. The opposite hand grabs the backside of the elbow and presses it towards the opposite shoulder.

\section{- Sleepers stretch}

The patient is lying on the painful side. The arm and elbow are flexed 90 degrees, the elbow pointing forwards. A gentle pressure downwards is applied on the wrist by the opposite hand.

Ad strengthening exercises (rotatorcuff and scapular muscles):

4 rows of exercises, with a decreasing number of repetition and increasing weight over time. Pause of $2-3 \mathrm{~min}$ between each rows. The exercises are performed slowly, with $3 \mathrm{~s}$ for the concentric fase, and $3 \mathrm{~s}$ for the eccentric fase ( $6 \mathrm{~s}$ per repetition), 3 times per week.

- 1st. week: 15 repetitions with maximal weight (RM)

- Week 2-3: 12 RM

- Week 4-5: 10 RM

- Week 6-8: 8 RM

- Week 9-12: 6 RM

Ad stretching exercises: the position is maintained for 60 seconds and repeated 4 times. 


\section{Home based exercise (HTR)}

- Posterior shoulder stretch $30 \mathrm{~s} 5$ times / day: the patients lifted their arm 90 degrees and pulled the elbow towards the opposite shoulder with their free hand.

- Pectoralis minor stretch $30 \mathrm{~s} 5$ times / day: the patients placed each hand at shoulder height on adjacent walls of a corner and leant forward

- Upper trapezius relaxation exercise 5 times / day: the patients where instructed to abduct their arms without a shrugging the shoulders.

- Serratus anterior strengthening exercise: the patients lifted a weight vertically from a supine position and protraction of the scapulas.

- Humeral external rotation with a rubber band and the arm at the side of the body.

- Humeral external rotation with a rubber band and the arm at 90 degrees abduction.

Ad weight and rubber band training:

1. 10 repetitions $\times 3$ per day the 1 st. week.

2. 15 repetitioner $\times 3$ per day the 2 nd. week.

3. 20 repetitioner $\times 3$ per day the 3 rd. week.

4. On following weeks, the patients were instructed to repeat the repetition sessions with heavier weight, and rubber band with increased resistance.

Both the STR and the HTR groups exercised for 3 months, and were suggested to continue exercising on their own afterwards, in case of persisting symptoms.

\section{Compliance}

We defined 3 levels of compliance.

Level 1: full compliance, defined as performance of all planned training sessions.

Level 2: partial compliance, where only part of the planned exercises sessions were performed.

Level 3: no compliance.

Compliance was self-reported by the participants, and recorded at each visit.

\section{Evaluation}

Data were only registered for patients included in the study. Assessment at entrance was performed by the main author, and all subsequent clinical evaluations by the same clinical assistant, blinded to the group the patient had been allocated to. The primary author made all the ultrasound exams at study entrance, prior to randomisation. Clinical assessment was performed at inclusion, at 4,8 , and 12 weeks after training start, and 6 months after ended training according to the protocol.

At each visit, we registered if training had been performed according to the instructions (full training, partial training, no training at all).

\section{Effect-variables}

Primary effect variables

- CS (max. 100 points).

- SRQ ( $\max 100$ points).

For both CS and SRQ, higher scores indicated better outcome.

The CS assesses four aspects related to shoulder pathology; two subjective: pain and activities of daily living (ADL) and two objective: range of motion (ROM) and strength. The subjective components can receive up to 35 points and the objective 65 , resulting in a possible maximum total score of 100 points (best function). Pain and ADL are answered by the patient; ROM and strength require a physical evaluation by the examiner. In a systematic review [40] quality ratings reached a level of $75 \%$ or higher. Studies evaluating the content validity of the Constant-Murley score suggest that the description in the original publication is insufficient to accomplish standardization between centers and evaluators. Despite this limitation, the Constant-Murley score correlates strongly ( $>$ or $=0.70$ ) with shoulder-specific questionnaires, reaches acceptable benchmarks (rho >0.80) for its reliability coefficients, and is responsive (effect sizes and standardized response mean $>0.80$ ) for detecting improvement after intervention in a variety of shoulder pathologies.

The SRQ is a self-administered questionnaire designed to assess the severity of symptoms related to and the functional status of the shoulder. It includes domains of global assessment, pain, daily activities, recreational and athletic activities, work, satisfaction, and areas for improvement, some questions being rated more than others. It ranges between 17 and 100 (best score). It has been found to be valid, reliable, and responsive to clinical change [41].

Secondary effect variables

- Passive motion (flexion, abduction, internal and external rotation) was assessed with a goniometer.

- Active motion (flexion, abduction, internal and external rotation) was assessed with a goniometer.

- VAS (Visual Analogue Scale) on a $10 \mathrm{~cm}$ scale for each muscle test (full can test, empty can test, lift off 
test, resisted external rotation, palm-up test, Yergason's test). 0 indicates no pain, and 10 worst pain.

\section{Full can test}

The patient is seated or standing, holding their arm in $90^{\circ}$ of elevation in the scapular plane and the patient's thumb pointing up. The examiner then applies a downward force to the arm whilst the patient tries to resist it. The test is considered positive if the patient experiences pain in the arm.

\section{Empty can test}

The patient is seated or standing, holding their arm in $90^{\circ}$ of elevation in the scapular plane and the patient's thumb pointing down. The examiner then applies a downward force to the arm whilst the patient tries to resist it. The test is considered positive if the patient experiences pain in the arm.

\section{Lift off test}

The patient is standing and is asked to place their hand behind their back with the dorsum of the hand resting in the region of the lumbar spine. The examiner then applies force against the patients hand whilst the patient tries to lift his hand off the back, increasing internal rotation. The test is considered positive if the patient experiences pain in the arm.

\section{Resisted external rotation test}

The patient is seated or standing with their arm by the side, and the elbow flexed at 90 degrees. The examiner stabilizes the elbow against the patients body with one hand. The patient is then asked to turn its forearm outwards, against the resistance of the examiners hand placed on the dorsal side of the wrist. The test is considered positive if the patient experiences pain in the arm.

\section{Palm-up test}

The patient is seated or standing. The palm-up test was performed with the arm elevated anteriorly against resistance while the elbow was in extension and the forearm supinated. The test is considered positive if the patient experiences pain in the anterior and upper part of the arm.

\section{Yergason's test}

The patient is seated or standing. The patient's elbow was flexed at 90 degrees with the arm by the side and the forearm in pronation. The patient was then asked to perform an active supination against the resistance of the physician's hand placed on the wrist. The test is considered positive if the patient experiences pain in the bicipital groove.

$$
\text { - Neer's test (rated positive }=1 \text { and negative }=0 \text { ). }
$$

The examiner stabilized the patient's scapula with one hand, while passively flexing the patients arm with the other hand. If the patient reported pain in the arm in this position, the result of the test was considered to be positive

- Hawkins test (rated positive $=1$ and negative $=0$ ).

The patient's arm was raised in front of the body to $90^{\circ}$, and then into internal rotation by the examiner. The test was positive if it elicited arm pain.

\section{Ultrasound}

The integrity of the following structures was examined, and compared with the contralateral shoulder: caput longum bicipitis, the supraspinatus, infraspinatus and subscapularis tendons, and the sulcus bicipitalis and glenohumeral joint as regards to effusion. Tendons were evaluated as regards to thickening, as well as fibrillar disruption pointing at partial or full-thickness tear. Degeneration was defined as a tendon with a heterogenic ultrasound appearance, with loss of normal echogenic fibrillar appearance. These findings are associated with tendinopathy [42].

\section{Analysing strategy/statistics}

Results were analyzed by an external statistician, according to intention to treat principles. The last observation carried forward (LOCF) method was applied for missing data from dropouts.

An a priori power analysis was performed based on the clinical important difference and on the standard deviation (SD) for CS. These two quantities vary considerable in the literature. Based on previous data [43-45] a clinically relevant between-group difference of 10 and a SD of 15 were chosen for analysis. A significance level of $5 \%$ and a power of $90 \%$ resulted in a total sample size of 100 , i.e. 50 in each group. To minimize the risk of underpowering, a total of 126 patients were included in the study.

Descriptive statistics with mean and standard deviation were used to characterize the patients. The Student's t-test was used to compare intra-group and inter-group results, and the Chi-squared test to compare binary outcomes.

The statistical software package IBM SPSS Statistics v. 25 was used for analyses. 


\section{Results}

63 participants were assigned to either the STR or the HTR group and analysed. The analysis was by original assigned groups.

The male/female ratio was 33/30 in the STR group and $32 / 31$ in the HTR group.

The average age in the STR group was 61.7, and 60.3 years in group II $(p=0.60)$ (Table 1$)$.

With the exception of Full can test, which had a higher VAS score at baseline in the HTR group $(p=0.04)$, both groups were identical as regards to all other parameters (Table 1).

Table 1 Baseline characteristics

\begin{tabular}{|c|c|c|c|}
\hline & $\begin{array}{l}\text { STR Group } \\
n=63\end{array}$ & $\begin{array}{l}\text { HTR Group } \\
n=63\end{array}$ & $\mathbf{p}$ \\
\hline Age (mean $\pm S D$ ) & $61.7 \pm 13.4$ & $60.3 \pm 13.0$ & 0.56 \\
\hline Male/female (n) & $33 / 30$ & $32 / 31$ & - \\
\hline \multicolumn{4}{|l|}{ Employment status: } \\
\hline $\begin{array}{l}\text { Employed/unemployed/full time } \\
\text { sick leave/partial sick leave/retired } \\
\text { (n) }\end{array}$ & $33 / 2 / 2 / 0 / 26$ & $33 / 3 / 2 / 0 / 25$ & - \\
\hline \multicolumn{4}{|l|}{ Shoulder scores (mean \pm SD) } \\
\hline Constant Score (0-100) & $37.7 \pm 11.6$ & $36.3 \pm 9.7$ & 0.47 \\
\hline $\begin{array}{l}\text { Shoulder Rating Questionnaire } \\
(0-100)\end{array}$ & $54.1 \pm 14.5$ & $50.2 \pm 14.9$ & 0.14 \\
\hline \multicolumn{4}{|l|}{ Motion (degrees) } \\
\hline Passive flexion & $148.8 \pm 37.8$ & $141.6 \pm 42.0$ & 0.32 \\
\hline Passive abduction & $119.7 \pm 42.4$ & $109.0 \pm 40.1$ & 0.15 \\
\hline Passive outer rotation & $42.4 \pm 11.6$ & $43.1 \pm 9.5$ & 0.74 \\
\hline Passive inner rotation & $3.0 \pm 1.5$ & $3.1 \pm 1.6$ & 0.61 \\
\hline Active flexion & $145.1 \pm 39.4$ & $140.6 \pm 42.4$ & 0.54 \\
\hline Active abduction & $115.0 \pm 42.8$ & $107.6 \pm 40.3$ & 0.32 \\
\hline Active outer rotation & $42.4 \pm 11.6$ & $43.0 \pm 9.5$ & 0.77 \\
\hline Active inner rotation & $3.0 \pm 1.5$ & $3.2 \pm 1.6$ & 0.50 \\
\hline \multicolumn{4}{|l|}{ Muscle tests (VAS scale 0-10) } \\
\hline Full can test & $4.4 \pm 2.7$ & $5.4 \pm 2.9$ & 0.04 \\
\hline Empty can test & $4.7 \pm 2.7$ & $5.3 \pm 2.9$ & 0.19 \\
\hline Lift off test & $3.4 \pm 3.3$ & $4.4 \pm 3.3$ & 0.12 \\
\hline Resisted external rotation test & $3.1 \pm 2.9$ & $3.4 \pm 2.9$ & 0.61 \\
\hline Palm up test & $5.1 \pm 3.3$ & $5.0 \pm 3.1$ & 0.95 \\
\hline Yergasons test & $2.3 \pm 2.4$ & $2.0 \pm 2.4$ & 0.53 \\
\hline \multicolumn{4}{|l|}{ Ultrasonography (n) } \\
\hline Joint effusion, yes/no & $3 / 60$ & $1 / 62$ & - \\
\hline Sulcus effusion, yes/no & $17 / 46$ & $16 / 47$ & - \\
\hline \multicolumn{4}{|l|}{$\begin{array}{l}\text { Normal/oedema/partial lesion/ } \\
\text { total lesion/degeneration (n) }\end{array}$} \\
\hline Caput longum bicipitis & $56 / 7 / 0 / 0 / 0$ & $53 / 8 / 1 / 1 / 0$ & - \\
\hline Subscapularis & $51 / 3 / 0 / 0 / 9$ & $55 / 3 / 0 / 0 / 5$ & - \\
\hline Supraspinatus & $15 / 28 / 16 / 0 / 4$ & $15 / 31 / 14 / 0 / 3$ & - \\
\hline Infraspinatus & $60 / 1 / 1 / 0 / 1$ & $60 / 1 / 2 / 0 / 0$ & - \\
\hline
\end{tabular}

\section{Intra group results (Tables 2 and 4)}

- Within each group all parameters improved significantly between the 1 st

and the 4th visit where patients had trained for 3 months and completed training, and between the 1st and the 5 th visit, 6 months after training completion, during which participants were encouraged to continue training in case of persistent symptoms.

- Between the 1st. and 5th. visit, CS improved by 22.7 points for the STR group an

by 23.7 points for the HTR $(p=0.0001)$. The SRQ improved by 17.7 and 18.1 for the STR and the HTR groups respectively $(\mathrm{p}=0.0001)$. Range of motions improved in all directions, best for passive and active abduction, which improved with $26.9(p=0.03)$ and 30 degrees for the STR group $(p=0.0001)$ and with respectively 39.3 and 39.2 degrees for the HTR group $(\mathrm{p}=0.0001)$. Impingement tests normalised in both groups, best for the Neer's test, which became negative in $60 \%$ of the cases in both training regimens $(p<0.0001)$. Hawkins test normalised by $23 \%$ for the STR group $(p=0.05)$ and $22 \%$ for the HTR group $(p<0.02)$. Resistive tests all achieved statistical significance with regard to diminished pain, best for the full can test, and the palm-up test. VAS scores improved respectively for the STR end the HTR groups by 2.2 and 1.9 for the full can test $(\mathrm{p}=0.0001)$, and by 3.0 and 2.5 for the palm-up test $(\mathrm{p}=0.0001)$.

- Between the 4 th and the 5 th visit, there was significant improvement in CS,

and both passive and active internal rotation in both groups. SRQ improved significantly for the STR group, but not for the HTR.

\section{Inter group results (Tables 3 and 4)}

Neither between evaluation at inclusion and the 4th nor 5th visit, did we found any significant difference between the 2 groups, regardless of the variable considered.

The average improvements in CS and SRQ between visit 1 and 5 (and 4) may be considered not only statistically significant but also clinically meaningful in both groups although between group-differences were not observed [46]. This may be explained by spontaneous improvement and/or efficacy of both training regimens. 
Table 2 Changes in shoulder scores between visits

\begin{tabular}{|c|c|c|c|c|c|c|c|c|c|c|c|c|}
\hline \multirow[t]{4}{*}{ Variable } & $\begin{array}{l}\text { Visit } \\
4 \text { vs. } 1\end{array}$ & p & $\begin{array}{l}\text { Visit } \\
4 \text { vs. } 1\end{array}$ & $\mathrm{p}$ & $\begin{array}{l}\text { Visit } \\
5 \text { vs. } 1\end{array}$ & p & $\begin{array}{l}\text { Visit } \\
5 \text { vs. } 1\end{array}$ & $\mathrm{p}$ & $\begin{array}{l}\text { Visit } \\
5 \text { vs. } 4\end{array}$ & p & $\begin{array}{l}\text { Visit } \\
5 \text { vs. } 4\end{array}$ & p \\
\hline & STR Group & & HTR Group & & STR Group & & HTR Group & & STR Group & & HTR Group & \\
\hline & $n=63$ & & $n=63$ & & $n=63$ & & $n=63$ & & $n=63$ & & $n=63$ & \\
\hline & Mean \pm SD & & Mean \pm SD & & Mean \pm SD & & Mean \pm SD & & Mean \pm SD & & Mean \pm SD & \\
\hline \multicolumn{13}{|c|}{ Shoulder scores } \\
\hline CS & $16.0 \pm 15.8$ & 0.0001 & $19.7 \pm 16.7$ & 0.0001 & $22.7 \pm 20.5$ & 0.0001 & $23.7 \pm 18.2$ & 0.0001 & $6.7 \pm 11.9$ & 0.0001 & $4.0 \pm 11.6$ & 0.008 \\
\hline SRQ & $11.7 \pm 4.2$ & 0.0001 & $16.4 \pm 18.1$ & 0.0001 & $17.7 \pm 19.5$ & 0.0001 & $18.1 \pm 18.1$ & 0.0001 & $6.0 \pm 12.0$ & 0.0001 & $1.6 \pm 10.3$ & 0.21 \\
\hline \multicolumn{13}{|c|}{ Passive Motion (degrees) } \\
\hline Flexion & $12.8 \pm 35.7$ & 0.0001 & $16.9 \pm 38.3$ & 0.0001 & $15.1 \pm 38.6$ & 0.038 & $21.4 \pm 41.6$ & 0.0001 & $2.26 \pm 24.6$ & 0.72 & $4.5 \pm 22.0$ & 0.09 \\
\hline Abduction & $21.2 \pm 38.1$ & 0.0001 & $33.4 \pm 44.4$ & 0.0001 & $26.9 \pm 39.4$ & 0.003 & $39.3 \pm 46.6$ & 0.0001 & $5.9 \pm 32.0$ & 0.15 & $5.9 \pm 31.7$ & 0.14 \\
\hline Ext. rotation & $4.6 \pm 9.2$ & 0.0001 & $3.2 \pm 9.36$ & 0.0001 & $5.2 \pm 9.0$ & 0.0001 & $3.8 \pm 9.7$ & 0.0020 & $0.65 \pm 4.5$ & 0.26 & $0.63 \pm 2.9$ & 0.10 \\
\hline Int. rotation & $1.1 \pm 1.5$ & 0.0001 & $1.0 \pm 1.5$ & 0.0001 & $1.5 \pm 1.6$ & 0.0001 & $1.3 \pm 1.8$ & 0.0001 & $0.37 \pm 1.1$ & 0.01 & $0.30 \pm 1.0$ & 0.02 \\
\hline \multicolumn{13}{|c|}{ Active Motion (degrees) } \\
\hline Flexion & $13.9 \pm 33.7$ & 0.0001 & $17.7 \pm 39.1$ & 0.0001 & $16.5 \pm 39.3$ & 0.002 & $21.8 \pm 42.7$ & 0.0001 & $2.7 \pm 26.9$ & 0.44 & $4.06 \pm 20.7$ & 0.12 \\
\hline Abduction & $24.1 \pm 36.4$ & 0.0001 & $34.0 \pm 44.8$ & 0.0001 & $30.0 \pm 41.6$ & 0.0001 & $39.2 \pm 47.7$ & 0.0001 & $5.9 \pm 31.9$ & 0.15 & $5.2 \pm 31.5$ & 0.19 \\
\hline Ext. rotation & $4.6 \pm 9.2$ & 0.0001 & $3.3 \pm 9.4$ & 0.007 & $5.2 \pm 9.0$ & 0.0001 & $3.9 \pm 9.7$ & 0.002 & $0.65 \pm 4.5$ & 0.26 & $0.63 \pm 2.9$ & 0.09 \\
\hline Int. rotation & $1.0 \pm 1.5$ & 0.0001 & $0.97 \pm 1.6$ & 0.0001 & $1.4 \pm 1.6$ & 0.0001 & $1.3 \pm 1.9$ & 0.0001 & $0.37 \pm 1.1$ & 0.01 & $0.30 \pm 1.0$ & 0.02 \\
\hline \multicolumn{13}{|c|}{ Muscle tests (VAS scale) } \\
\hline Full can & $-1.7 \pm 2.7$ & 0.0001 & $-1.9 \pm 3.4$ & 0.0001 & $-2.2 \pm 3.0$ & 0.0001 & $-1.9 \pm 3.8$ & 0.0001 & $-0.49 \pm 2.0$ & 0.06 & $-0.04 \pm 2.5$ & 0.90 \\
\hline Empty can & $-1.9 \pm 2.5$ & 0.0001 & $-1.5 \pm 2.8$ & 0.0001 & $-1.9 \pm 2.9$ & 0.0001 & $-1.5 \pm 3.2$ & 0.0001 & $-0.02 \pm 1.6$ & 0.93 & $-0.04 \pm 1.8$ & 0.87 \\
\hline Lift off & $-0.81 \pm 2.7$ & 0.02 & $-1.5 \pm 3.4$ & 0.0001 & $-1.0 \pm 3.6$ & 0.028 & $-1.7 \pm 3.5$ & 0.0001 & $-0.22 \pm 2.4$ & 0.46 & $-0.02 \pm 1.5$ & 0.30 \\
\hline Ext. rotation & $-1.4 \pm 2.4$ & 0.0001 & $-1.40 \pm 2.0$ & 0.0001 & $-1.3 \pm 2.7$ & 0.0001 & $-1.6 \pm 2.2$ & 0.0001 & $0.07 \pm 1.5$ & 0.72 & $-0.20 \pm 1.2$ & 0.19 \\
\hline Palm up & $-2.8 \pm 3.0$ & 0.0001 & $-2.3 \pm 3.1$ & 0.0001 & $-3.0 \pm 3.1$ & 0.0001 & $-2.5 \pm 3.3$ & 0.0001 & $-0.15 \pm 1.9$ & 0.51 & $-0.21 \pm 1.9$ & 0.39 \\
\hline Yergason & $-0.92 \pm 2,3$ & 0.003 & $-0.72 \pm 2.0$ & 0.007 & $-0.90 \pm 2.8$ & 0.02 & $-0.81 \pm 2$ & 0.002 & $-0.09 \pm 1,4$ & 0.62 & $0.02 \pm 1.3$ & 0.90 \\
\hline
\end{tabular}

\section{Drop out rate (Table 5)}

At visit 4, 13 patients had dropped out in the STR group and 19 in the HTR group $(p=0.2)$. The corresponding numbers for visit 5 were 21 and $27(p=0.3)$. Identifiable reasons for dropout were failure to perform the exercises because of pain, lack of time, or concomitant disease (Table 6).

\section{Compliance}

We calculated a mean level of compliance, which was 1.38 for the STR group and 1.51 for the HTR group. The difference between the groups was non-significant $(p=0.093)$.

\section{Ultrasonography}

No difference was found at baseline between the groups (Table 1).

\section{Discussion}

Our study showed that the effect of a comprehensive training regimen including heavy training principles and consisting of 6 motion, 8 strengths and 4 stretching exercises performed under supervision of a physiotherapist was not better than a much simpler home-based program offering 1 motion, 3 progressive strength, and 2 stretching exercises. There was within-group improvement for almost all parameters, but no between-group differences.

\section{Supervised versus home-based training}

The benefit of adding supervision to a training regimen including strengthening exercises is poorly documented. In a newly systematic review, supervised training and self training resulted in equal improvement of pain and function, and larger improvement than no training for patients with subacromial pain syndrome [6].

In another recent systematic review, supervised physical therapy and home-based progressive shoulder strengthening and stretching exercises for the rotatorcuff and scapular muscles were equally effective in patients with SIS treated conservatively [7].

It seems that supervision yields better compliance and more correct performance of the exercises [47-49]. However, in a study of patients who had undergone rotator cuff repair, no statistical differences were found between an exercise program under the supervision of a physiotherapist and a standardized home-based exercise 
Table 3 Inter-group differences (STR vs. HTR group) in score changes between visits

\begin{tabular}{|c|c|c|c|c|c|c|c|c|c|}
\hline \multirow[t]{3}{*}{ Variable } & $\begin{array}{l}\text { Inter-group } \\
\text { difference }\end{array}$ & SE & $\mathrm{p}$ & $\begin{array}{l}\text { Inter-group } \\
\text { difference }\end{array}$ & SE & $\mathbf{p}$ & $\begin{array}{l}\text { Inter-group } \\
\text { difference }\end{array}$ & SE & $\mathrm{p}$ \\
\hline & \multicolumn{3}{|l|}{ Mean } & \multicolumn{3}{|l|}{ Mean } & \multicolumn{3}{|l|}{ Mean } \\
\hline & \multicolumn{3}{|l|}{ Visit 4 vs. 1} & \multicolumn{3}{|l|}{ Visit 5 vs. 1} & \multicolumn{3}{|l|}{ Visit 5 vs. 4} \\
\hline \multicolumn{10}{|l|}{ Shoulder Scores } \\
\hline Constant score & -3.7 & 2.9 & 0.20 & -1.0 & 3.5 & 0.77 & 2.7 & 2.1 & 0.20 \\
\hline $\mathrm{SRQ}$ & -4.8 & 2.9 & 0.10 & -0.42 & 3.4 & 0.90 & 4.4 & 2.0 & 0.03 \\
\hline \multicolumn{10}{|c|}{ Passive motion (degrees) } \\
\hline Flexion & -4.1 & 6.6 & 0.54 & -6.3 & 7.16 & 0.38 & -2.3 & 4.1 & 0.58 \\
\hline Abduction & -12.3 & 7.4 & 0.10 & -12.4 & 7.7 & 0.11 & -0.05 & 5.7 & 0.99 \\
\hline Ext. rotation & 1.39 & 1.65 & 0.400 & 1.41 & 1.7 & 0.40 & 0.02 & 0.70 & 0.98 \\
\hline Int. rotation & 0.03 & 0.27 & 0.90 & 0.11 & 0.31 & 0.73 & 0.07 & 0.19 & 0.70 \\
\hline \multicolumn{10}{|c|}{ Active motion (degrees) } \\
\hline Flexion & -3.9 & 6.5 & 0.55 & -5.3 & 7.3 & 0.47 & -1.4 & 4.2 & 0.74 \\
\hline Abduction & -9.9 & 67.3 & 0.18 & -9.2 & 8.0 & 0.25 & 0.65 & 5.6 & 0.91 \\
\hline Ext. rotation & 1.32 & 1.6 & 0.43 & 1.34 & 1.7 & 0.42 & 0.02 & 1.7 & 0.98 \\
\hline Int. rotation & 0.05 & 0.27 & 0.86 & 0.12 & 0.31 & 0.70 & 0.07 & 0.19 & 0.70 \\
\hline \multicolumn{10}{|c|}{ Muscle tests (VAS scale) } \\
\hline Full can & 0.23 & 0.55 & 0.67 & -0.21 & 0.60 & 0.73 & -0.45 & 0.41 & 0.28 \\
\hline Empty can & -0.36 & 0.48 & 0.45 & -0.34 & 0.54 & 0.53 & .02 & 0.30 & 0.95 \\
\hline Lift off & 0.65 & 0.55 & 0.24 & 0.62 & 0.63 & 0.33 & -0.03 & 0.35 & 0.93 \\
\hline Ext. rotation & 0.00 & 0.39 & 0.99 & 0.27 & 0.43 & 0.54 & 0.27 & 0.24 & 0.26 \\
\hline Palm up & 0.05 & 0.55 & .878 & -0.43 & 0.57 & 0.46 & -0.48 & 0.33 & 0.38 \\
\hline Yergason & -0.21 & 0.39 & 0.60 & -0.09 & 0.43 & 0.83 & 0.11 & 0.24 & 0.65 \\
\hline
\end{tabular}

SE standard error of difference

Table 4 Neer's and Hawkins test for impingement results (positive or negative) with p-values for intra-group between-visits differences and inter-group differences

\begin{tabular}{|c|c|c|c|c|c|c|c|c|c|c|c|c|c|c|c|}
\hline & STR G & oup (n & $=63)$ & & & & HTR C & oup (n & $=63)$ & & & & STR vs. & HTR gro & \\
\hline & Visit & & & & & & Visit & & & & & & Visit & & \\
\hline & 1 & 4 & 5 & 4 vs. 1 & 5 vs. 1 & 5 vs. 4 & 1 & 4 & 5 & 4 vs. 1 & 5 vs. 1 & 5 vs. 4 & 1 vs. 1 & 4 vs. 4 & 5 vs. 5 \\
\hline & Positi & e:Nega & ive & $p$ & & & Positi & e:Nega & ive & $p$ & & & $p$ & & \\
\hline & $\mathrm{n}: \mathrm{n}$ & & & & & & $\mathrm{n}: \mathrm{n}$ & & & & & & & & \\
\hline Neer's & $51: 11$ & $17: 45$ & $14: 48$ & $<0.0001$ & $<0.0001$ & 0.55 & $56: 8$ & $21: 43$ & $18: 46$ & $<0.0001$ & $<0.0001$ & 0.45 & 0.41 & 0.51 & 0.48 \\
\hline Hawkins & $41: 21$ & $37: 25$ & $30: 32$ & 0.34 & 0.11 & 0.10 & $45: 19$ & $33: 31$ & $31: 33$ & $<0.03$ & $<0.008$ & 0.88 & 0.10 & 0.34 & 1.00 \\
\hline
\end{tabular}

Intra-group comparison of proportions was performed using McNemar's test

Inter-group comparison of proportions was performed using the "N-1" Chi-squared test

program regarding pain, functional status, quality of life and depression status [50]. Both programs included active and strengthening exercises. Supervised rehabilitation with strengthening exercises of the rotator cuff and scapula stabilizers seems to be superior to home exercises focusing on mobility for improving shoulder function after arthroscopic acromioplasty [51]. Thus, the type of exercises offered seems to be important.

\section{The heavy training component}

The poor result of the addition of heavy training, is in accordance with other studies [51-54]. 
Table 5 Dropouts

\begin{tabular}{llll}
\hline & $\begin{array}{l}\text { STR Group } \\
\mathbf{n = 6 3}\end{array}$ & $\begin{array}{l}\text { HTR Group } \\
\mathbf{n}=\mathbf{6 3}\end{array}$ & $\mathbf{p}$ \\
\hline Dropout rate at & & & \\
Visit 2 & 5 & 8 & 0.418 \\
Visit 3 & 8 & 12 & 0.335 \\
Visit 4 & 13 & 19 & 0.218 \\
Visit 5 & 21 & 27 & 0.274
\end{tabular}

"N-1" Chi-squared test as recommended by Campbell (2007) and Richardson (2011)

Campbell I (2007) Chi-squared and Fisher-Irwin tests of two-by-two tables with small sample recommendations. Statistics in Medicine 26:3661-3675. PubMed

Richardson JTE (2011) The analysis of $2 \times 2$ contingency tables - Yet again.

Statistics in Medicine 30:890. PubMed

Table 6 Reasons for discontinuing the intervention (n)

\begin{tabular}{lll}
\hline Training group & STR & HTR \\
\hline Non-compliant to training & 2 & 2 \\
Steroid injection & 1 & \\
New job & 1 & 3 \\
Pain & 12 & 17 \\
Did not show up & 1 & 2 \\
Did not have the time & & 1 \\
Preferred other kind of training & 1 & \\
Hand surgery & 1 & 1 \\
Moved & & 1 \\
Parkinsonism & 1 & 27 \\
Treatment with prednisolone & 1 & \\
Frozen shoulder & 21 & \\
Total &
\end{tabular}

Supervised strengthening program has been shown to be superior compared to home-based unresisted movement training [51], but high load training may not be superior to low load training. Thus, in a study of 100 patients, Ingwersen et al., found no difference between a group who trained according to the principles of Heavy Slow resistance training and a group who were rehabilitated with low level exercises [53]. In another study, Maenhout et al., added heavy eccentric training to a traditional rotator cuff strengthening program, resulting in higher isometric strength at 90 degrees of abduction, but without any effect on pain or function [54]. In a systematic review [52], it was found that for persistent subacromial pain, supervised and home-based strengthening leads to similar outcomes as surgery and that home-based heavy load eccentric training does not add benefits to home-based rotator cuff strengthening and physiotherapy.

HSRT has been shown to reduce pain in Achilles and patellar tendinopathy [21]. As training of the rotator cuff tendons is a substantial part of SAIS rehabilitation, it would therefore make sense, that HSRT would yield good results in that field as well. The results of our and other studies seem to contradict this assumption. It may be, that tendons involved in multidirectional actions, as for the rotator cuff, present different challenges to rehabilitation, than patellar and Achilles tendons, which mainly have a unidirectional function.

\section{The comprehensive regimen}

Considering the complicated biomechanics of the shoulder, we were expecting that our program, consisting of a comprehensive set of exercises with focus on restoring mobility and strength of the scapular muscles and rotator cuff tendons, would reveal superior to the simpler program designed by Ludewig et al. [5]. But our population had an average age of over 60 years, and even though sport attendance wasn't registered, we speculate that a younger and more sporty group, might have performed better in the STR group. This theory is supported by the observations of our physiotherapist, who found that in the supervised group, several patients had difficulties performing the exercises correctly. Unfortunately, this wasn't assessed, but proper execution of instructed exercises has been reported as challenging, and in another Danish study, only a quarter of the patients performed their home-based shoulder abduction exercise correctly when evaluated 2 weeks after the instruction by a physiotherapist [47].

\section{Drop-out rate}

Out of 48 patients lost to follow-up at visit 5, 12 from the STR group and 17 from the HTR group just didn't show up, and 2 from the HTR group stopped doing the exercises. Other causes of dropout were failure to perform the exercises because of pain, lack of time, or concomitant disease. Participants were only assessed with ultrasonography of their shoulder at entrance. Any worsening was assessed clinically, and resulted in withdrawal from the study in a few cases. Two participants withdrew because they developed a frozen shoulder, and 6 because of pain. After withdrawal, ultrasonography was performed. In no cases did we detect any worsening of the rotatorcuff condition (unpublished data). We speculate that the duration of the trial, with the last follow-up being 9 months after inclusion might have been a substantial cause of dropout. We were unfortunately unable to get in touch with the participants that didn't show up, and can therefore not offer any other explanation.

\section{Compliance}

Two important issues for the success of exercise therapy are correct performance of prescribed exercise and 
compliance, which is reported as variable, but often low, especially for home-based exercise regimens. Compliance for home based exercises was found to be as low as $29 \%$ in a Danish study on helicopter pilots [48] and as high as $86 \%$ in a study comparing eccentric with concentric supraspinatus training [36] even though supervised training regimens often have greater compliance than home based exercises [49]. In our study, contrary to our expectations, compliance defined as completion of the exercise regimens, was without significant difference between the two groups. We find it possible, that adherence to the training protocols, has been enhanced by the prospect of meeting up to assessment every month.

\section{Baseline characteristics}

This was a randomised controlled trial. Consequently, the study groups were not "matched". But it turned out that the two groups were comparable regarding most baseline characteristics shown in Table 1, with the exception of Full can test, which had a higher VAS score at baseline in group II $(p=0.04)$. We find it unlikely though, that this sole baseline characteristic would account for the lack of between-group difference in all end-result parameters. As this was a randomised controlled trial, spontaneous improvers are taken into account when comparing the groups.

\section{Study limitations/ possible flaws}

The diagnosis of SAIS is a challenge [55-57]. Patients in the present study fulfilled criteria that are accepted as reliable and accurate [24]. Other papers investigating training regimen for rotator cuff elicited pain and function reduction may have operated with different definitions [53], making direct comparison difficult. Ultrasonography at entrance, revealed edema, partial lesions or pathological findings in the supraspinatus tendon in all but 15 participants in each group. These 15 participants may well have had a rotator cuff problem anyway, as ultrasonography, despite its usefulness in shoulder pathology, can show normal findings in patients with a clinical rotator syndrome [58]. We therefore believe, that the risk of having included patients with different pathologies is small.

Several patients refused to enter our project, due to intense shoulder pain. We did not include steroid injection as an exclusion criterion in this trial in order to mimic clinical daily practice as much as possible. When needed, due to pain, patients were therefore offered between 1 and 3 injections with approximately 4 weeks interval before they were re-invited to participate in the trial. This approach may have resulted in a biased population as patients who had experienced severe pain for a long time may have preferred standard physiotherapy instead of challenging exercises in a clinical trial. This possibility is emphasized by the fact that none of the patients that required more than 1 steroid injection accepted to enter the study (data not registered).

Recruitment of patients proved particularly difficult, which is why it took us about 5 years to complete the study. The reason is unclear as these patients were not characterized or registered. Severe pain, lack of time, transportation time etc. may have contributed. Consequently, the patient sample studied may not be completely representative for SAIS patients in general.

For obvious reason, neither the patients nor the physiotherapist were blinded to the exercise regimen. Most of the patients wished to participate in the STR and expressed disappointment when randomised to HTR. Theoretically, this could affect the results negatively in the control group, However, any significant difference in favor of STR failed to be shown.

\section{Consideration for future research - future exercise programs}

One important consideration, when offering rehabilitation to a patient, is how to structure the program. In addition to having proper exercises, a rehabilitation program ought to promote good compliance with regards to correct exercise performance, amount of time spent on training in each training session and the duration of training over time. All of these training elements represent a challenge. Mobile Phone Text Messaging as Reminders of Home Exercises may be effective [59].

But low compliance may be due to other factors. In a Danish study, lower adherence to a 10-week exercise program of 2 or 12 min' duration, performed 5 times a week, was predicted by poorer psychosocial work environment and lower exercise self-efficacy. Interestingly, a longer exercise program was not associated with lower adherence [60]. Future research ought to focus on how to motivate this group for better training compliance.

It seems probable, that a more tailored program could boost the effect of training. An approach to more individualised programs could include longer and more challenging programs for patients used to recreational or professional sport activities as these patients have high requirements for shoulder function and may find homebased exercise with focus on repetition and endurance tedious. Conversely, simpler home-based exercises might be offered to patients with low physical activity with no incentive to perform challenging programs.

New techniques may be added, to structure the best functional rehabilitation programs. Thus, in a study using 3D-measurement techniques, magnetic resonance images were coupled with shoulder during exercises. The study showed how the glenohumeral joint, the labrum and the 
subacromial space were affected during the different exercises. That kind of knowledge may contribute to the development of better training regimens [61].

\section{Conclusion}

In this randomised controlled trial, we found no significant difference between a comprehensive supervised training regimen including heavy training principles, and a homebased training program.

\begin{abstract}
Abbreviations
CS: Constant score; HSRT: Heavy slow resistance training; HTR: Home-based regimen; LOCF: Last observation carried forward; LT: Lower trapezius; MT: Middle trapezius; ROM: Range of motion; SA: Serratus anterior; SIS: Subacromial impingement syndrome; SRQ: Shoulder rating questionnaire; STR: Supervised training regimen; UT: Upper trapezius; VAS: Visual analogue scale.
\end{abstract}

\section{Acknowledgments}

We thank Physiotherapist Anja Rosendahl, for providing training guidance to the participants.

\section{Authors' contributions}

All authors contributed to the study conception and design. Material preparation, data collection and analysis were performed by Pierre Schydlowsky, Marcin Szkudlarek and Ole Rintek Madsen. Pierre Schydlowsky generated the cards used for random allocation, enrolled participants, and assigned participants to interventions. The first draft of the manuscript was written by Pierre Schydlowsky and all authors commented on previous versions of the manuscript. All authors read and approved the final manuscript.

\section{Funding}

The project has been funded by the Danish Fund for the Professional Development of Specialist Clinics (Fonden for Faglig Udvikling af Speciallægepraksis).

\section{Availability of data and materials}

All data and materials, as well as software applications, support our claims and comply with filed standards. The datasets used and/or analysed during the current study are available from the corresponding author on reasonable request.

\section{Declarations}

\section{Ethics approval and consent to participate}

The trial was approved by the Ethics Committee of the Capital Region of Denmark on April 24th. 2013, protocol nr. H-4-2013-030.

Informed consent was obtained by all participants, in accordance with the 1964 declaration of Helsinki and its later amendments.

\section{Consent for publication}

Not Applicable.

\section{Competing interests}

None.

\section{Author details}

${ }^{1}$ Reumatologiklinikken i Værløse, Bymidten 11,1, 3500 Værløse, Denmark. ${ }^{2}$ Department of Rheumatology, Zealand University Hospital at Køge, Køge, Denmark. ${ }^{3}$ Institute of Clinical Medicine, University of Copenhagen, Copenhagen, Denmark. ${ }^{4}$ Department of Rheumatology and Spine Diseases, Gentofte University Hospital and Rigshospitalet, DK-2900 Hellerup, Denmark.

Received: 27 June 2021 Accepted: 17 December 2021 Published online: 15 January 2022

\section{References}

1. van der Windt DA, Koes BW, de Jong BA, Bouter LM. Shoulder disorders in general practice: incidence, patient characteristics, and management. Ann Rheum Dis. 1995;54:959-64. https://doi.org/10.1136/ard.54.12.959.

2. Walker-Bone K, Palmer KT, Reading I, Coggon D, Cooper C. Prevalence and impact of musculoskeletal disorders of the upper limb in the general population. Arthritis Rheum. 2004;51:642-51. https://doi.org/10.1002/art. 20535.

3. Perry SM, Getz CL, Soslowsky LJ. After rotator cuff tears, the remaining (intact) tendons are mechanically altered. J Shoulder Elb Surg. 2009;18:52-7. https://doi.org/10.1016/j.jse.2008.07.003.

4. Haahr JP, Østergaard S, Dalsgaard J, Norup K, Frost P, Lausen S, et al. Exercises versus arthroscopic decompression in patients with subacromial impingement: a randomised, controlled study in 90 cases with a one year follow-up. Ann Rheum Dis. 2005;64:760-4. https://doi.org/10.1136/ard. 2004.021188.

5. Ludewig PM, Borstad JD. Effects of a home exercise programme on shoulder pain and functional status in construction workers. Occup Environ Med. 2003;60:841-9. https://doi.org/10.1136/oem.60.11.841.

6. Liaghat B, Ussing A, Petersen BM, Andersen HK, Barfod KW, Jensen MB, Hoegh M, Tarp S, Juul-Kristensen B, Brorson S. Supervised training compared with no training or self-training in patients with subacromial pain syndrome: a systematic review and Meta-analysis. Arch Phys med Rehabil. 2021;28:S0003-9993(21)00318-X. doi: https://doi.org/10.1016/j.apmr.2021. 03.027. Online ahead of print.

7. Gutiérrez-Espinoza H, Araya-Quintanilla F, Cereceda-Muriel C, ÁlvarezBueno C, Martínez-Vizcaíno V, Cavero-Redondo I. Effect of supervised physiotherapy versus home exercise program in patients with subacromial impingement syndrome: a systematic review and meta-analysis. Phys Ther Sport. 2020;41:34-42. https://doi.org/10.1016/j.ptsp.2019.11. 003 Epub 2019 Nov 6.

8. Larsson R, Bernhardsson S, Nordeman L. Effects of eccentric exercise in patients with subacromial impingement syndrome: a systematic review and meta-analysis. BMC Musculoskelet Disord. 2019;20(1):446. https://doi. org/10.1186/s12891-019-2796-5.

9. Kuhn JE. Exercise in the treatment of rotatorcuff impingement: a systematic review and a synthesized evidence-based rehabilitation protocol. J Shoulder Elb Surg. 2009;18:138-60. https://doi.org/10.1016/j.jse.2008.06. 004.

10. Björnsson Hallgren $\mathrm{HC}$, Adolfsson LE, Johansson K, Öberg B, Peterson A, Holmgren TM. Specific exercises for subacromial pain. Acta Orthop. 2017;88:600-5. https://doi.org/10.1080/17453674.2017.1364069.

11. Holmgren T, Björnsson Hallgren H, Öberg B, Adolfsson L, Johansson K. Effect of specific exercise strategy on need for surgery in patients with subacromial impingement syndrome: randomised controlled study. BMJ. 2012;20(344):e787. https://doi.org/10.1136/bmj.e787.

12. Cools AM, Dewitte V, Lanszweert F, Notebaert D, Roets A, Soetens B, et al. Rehabilitation of scapular muscle balance: which exercises to prescribe? Am J Sports Med. 2007;35:1744-51. https://doi.org/10.1177/0363546507 303560.

13. Cools AM, Struyf F, De Mey K, Maenhout A, Castelein B, Cagnie B. Rehabilitation of scapular dyskinesis: from the office worker to the elite overhead athlete. Br J Sports Med. 2014;48:692-7. https://doi.org/10.1136/bjspo rts-2013-092148.

14. Michener LA, Sharma S, Cools AM, Timmons MK. Relative scapular muscle activity ratios are altered in subacromial pain syndrome. J Shoulder Elb Surg. 2016;25:1861-7. https://doi.org/10.1016/j.jse.2016.04.010.

15. Maenhout A, Van Eessel V, Van Dyck L, Vanraes A, Cools A. Quantifying acromiohumeral distance in overhead athletes with glenohumeral internal rotation loss and the influence of a stretching program. Am J Sports Med. 2012;40:2105-12. https://doi.org/10.1177/0363546512454530.

16. Tyler TF, Nicholas SJ, Lee SJ, Mullaney M, McHugh MP. Correction of posterior shoulder tightness is associated with symptom resolution in patients with internal impingement. Am J Sports Med. 2010;38:114-9. https://doi. org/10.1177/0363546509346050.

17. Desmeules F, Boudreault J, Dionne CE, Frémont P, Lowry V, MacDermid JC, et al. Efficacy of exercise therapy in workers with rotator cuff tendinopathy: a systematic review. J Occup Health. 2016;58:389-403. https://doi. org/10.1539/joh.15-0103-RA.

18. Tahran Ö, Yeşilyaprak SS. Effects of modified posterior shoulder stretching exercises on shoulder mobility, pain, and dysfunction in patients with 
subacromial impingement syndrome. Sports Health. 2020;12:139-48, https://doi.org/10.1177/1941738119900532 Epub 2020 Feb 4.

19. Turgut E, Duzgun I, Baltaci G. Stretching exercises for subacromial impingement syndrome: effects of 6-week program on shoulder tightness, pain, and disability status. J Sport Rehabil. 2018;27:132-7. https:// doi.org/10.1123/jsr.2016-0182 Epub 2018 Mar 8.

20. Gutiérrez-Espinoza H, Araya-Quintanilla F, Gutiérrez-Monclus R, RíosRiquelme M, Álvarez-Bueno C, Martínez-Vizcaino V, et al. Does pectoralis minor stretching provide additional benefit over an exercise program in participants with subacromial pain syndrome? A randomized controlled trial. Musculoskelet Sci Pract. 2019;44:102052. https://doi.org/10.1016/j. msksp.2019.102052 Epub 2019 Aug 25.

21. Kongsgaard M, Kovanen V, Aagaard P, Doessing S, Hansen P, Laursen AH, et al. Corticosteroid injections, eccentric decline squat training and heavy slow resistance training in patellar tendinopathy. Scand J Med Sci Sports. 2009;19:790-802. https://doi.org/10.1111/j.1600-0838.2009.00949.x.

22. Eriksen CS, Svensson RB, Gylling AT, Couppé C, Magnusson SP, Kjaer M. Load magnitude affects patellar tendon mechanical properties but not collagen or collagen cross-linking after long-term strength training in older adults. BMC Geriatr. 2019;19:30. https://doi.org/10.1186/ s12877-019-1043-0.

23. Moseley JB Jr, Jobe FW, Pink M, Perry J, Tibone J. EMG analysis of the scapular muscles during a shoulder rehabilitation program. Am I Sports Med. 1992;20:128-34. https://doi.org/10.1177/036354659202000206.

24. Michener LA, Walsworth MK, Doukas WC, Murphy KP. Reliability and diagnostic accuracy of 5 physical examination tests and combination of clinical tests for subacromial impingement syndrome. Arch Phys Med Rehab. 2009;90:1898-903. https://doi.org/10.1016/j.apmr.2009.05.015.

25. Conroy DE, Hayes KW. The effect of joint mobilization as a component of comprehensive treatment for primary shoulder impingement syndrome. J Orthop Sports Phys Ther. 1998;28:3-14. https://doi.org/10.2519/jospt. 1998.28.1.3.

26. Walther M, Werner A, Stahlschmidt T, Woelfel R, Gohlke F. The subacromial impingement syndrome of the shoulder treated by conventional physiotherapy, self-training, and a shoulder brace: results of a prospective, randomized study. J Shoulder Elb Surg. 2004;13:417-23. https://doi.org/ 10.1016/j.jse.2004.02.002.

27. Ellsworth AA, Mullaney M, Tyler TF, Malachy McHugh M, Nicholas S. Electromyography of selected shoulder musculature during un-weighted and weighted pendulum exercises. N Am J Sports Phys Ther. 2006;1:73-9.

28. Murphy CA, McDermott WJ, Petersen RK, Johnson SE, Baxter SA. Electromyographic analysis of the rotator cuff in postoperative shoulder patients during passive rehabilitation exercise. J Shoulder Elb Surg. 2013;22:102-7. https://doi.org/10.1016/j.jse.2012.01.021 Epub 2012 May 3.

29. Cunningham G, Charbonnier C, Lädermann A, Chagué S, Sonnabend DH. Shoulder motion analysis during Codman pendulum exercises. Arthrosc Sports Med Rehabil. 2020;(2):e333-9. https://doi.org/10.1016/j.asmr.2020. 04.013 eCollection 2020 Aug.

30. Castelein B, Cools A, Parlevliet T, Cagnie B. The influence of induced shoulder muscle pain on rotator cuff and scapulothoracic muscle activity during elevation of the arm. J Shoulder Elb Surg. 2017;26:497-505. https://doi.org/10.1016/j.jse.2016.09.005.

31. Chaconas EJ, Kolber MJ, Hanney WJ, Daugherty ML, Wilson SH, Sheets C. Shoulder external rotator eccentric training versus general shoulder exercise for subacromial pain syndrome: a randomised controlled trial. Int J Sports Phys Ther. 2017;12:1121-33. https://doi.org/10.26603/ijspt20171121.

32. Cha JY, Kim JH, Hong J, Choi YT, Kim MH, Cho JH, et al. A 12-week rehabilitation program improves body composition, pain sensation, and internal/external torques of baseball pitchers with shoulder impingement symptom. J Exerc Rehabil. 2014;10:35-44. https://doi.org/10.12965/ jer.140087.

33. Bak K, Magnusson SP. Shoulder strength and range of motion in symptomatic and pain-free elite swimmers. Am J Sports Med. 1997;25:454-9. https://doi.org/10.1177/036354659702500407.

34. Jobe FW, Pink M. Classification and treatment of shoulder dysfunction in the overhead athlete. J Orthop Sports Phys Ther. 1993;18:427-32. https:// doi.org/10.2519/jospt.1993.18.2.427.

35. Kibler WB, Sciascia AD, Uhl TL, Tambay N, Cunningham T. Electromyographic analysis of specific exercises for scapular control in early phases of shoulder rehabilitation. Am J Sports Med. 2008;36:1789-98. https://doi. org/10.1177/0363546508316281.
36. Bateman M, Adams N. A randomised controlled feasibility study investigating the use of eccentric and concentric strengthening exercises in the treatment of rotator cuff tendinopathy. SAGE Open Med. 2014;28(2):2050312113520151. https://doi.org/10.1177/2050312113520151.

37. Borstad JD, Ludewig PM. The effect of long versus short pectoralis minor resting length on scapular kinematics in healthy individuals. J Orthop Sports Phys Ther. 2005;35:227-38. https://doi.org/10.2519/jospt.2005.35.4.227.

38. Borstad JD, Ludewig PM. Comparison of three stretches for the pectoralis minor muscle. J Shoulder Elb Surg. 2006;15:324-30. https://doi.org/10. 1016/j.jse.2005.08.011.

39. Rosa DP, Borstad JD, Pogetti LS, Camargo PR. Effects of a stretching protocol for the pectoralis minor on muscle length, function, and scapular kinematics in individuals with and without shoulder pain. J Hand Ther. 2017;30:20-9. https://doi.org/10.1016/j.jht.2016.06.006.

40. Roy JS, MacDermid JC, Woodhouse LJ. A systematic review of the psychometric properties of the constant-Murley score. J Shoulder Elb Surg. 2010;19:157-64. https://doi.org/10.1016/j.jse.2009.04.008.

41. L'Insalata JC, Warren RF, CohenSB ADW, Peterson MG. A self-administered questionnaire for assessment of symptoms and function of the shoulder. J Bone Joint Surg Am. 1997;79:738-48.

42. Chang A, Miller TT. Imaging of tendons. Sports. Health. 2009;1:293-300. https://doi.org/10.1177/1941738109338361.

43. Kanto K, Lähdeoja T, Paavola M, Aronen P, Järvinen TLN, Jokihaara J, et al. Minimal important difference and patient acceptable symptom state for pain, constant-Murley score and simple shoulder test in patients with subacromial pain syndrome. BMC Med Res Methodol. 2021;21:45. https:// doi.org/10.1186/s12874-021-01241-w.

44. Henseler JF, Kolk A, Van der Zwaal P, et al. The minimal detectable change of the constant score in impingement, full-thickness tears, and massive rotator cuff tears. J Shoulder Elb Surg. 2015;24:376-8.

45. Dabija D, Jain NB. Minimal clinically important difference of shoulder outcome measures and diagnoses: a systematic review. Am J Phys Med Rehabil. 2019;98:671-6. https://doi.org/10.1097/PHM.0000000000001169.

46. Moser JS, Barker KL, Doll HA, Carr AJ. Comparison of two patient-based outcome measures for shoulder instability after nonoperative treatment. J Shoulder Elb Surg. 2008;17:886-92. https://doi.org/10.1016/j.jse.2008.05. 040 Epub 2008 Sep 10.

47. Faber M, Andersen MH, Sevel C, Thorborg K, Bandholm T, Rathleff M. The majority are not performing home-exercises correctly two weeks after their initial instruction-an assessor-blinded study. Peer J. 2015;21(3):e1102. https://doi.org/10.7717/peerj.1102.

48. Murray M, Lange B, Nørnberg BR, Søgaard K, Sjøgaard G. Self-administered physical exercise training as treatment of neck and shoulder pain among military helicopter pilots and crew: a randomized controlled trial. BMC Musculoskelet Disord. 2017;7(18):147. https://doi.org/10.1186/ s12891-017-1507-3.

49. Härkäpää K, Järvikoski A, Mellin G, Hurri H, Luoma J. Health locus of control beliefs and psychological distress as predictors for treatment outcome in low-back pain patients: results of a 3-month follow-up of a controlled intervention study. Pain. 1991;46:35-41. https://doi.org/10. 1016/0304-3959(91)90031-r.

50. Nihal B, Ali K, Semih A, Nuray A. Comparison of the results of supervised physiotherapy program and home-based exercise program in patients treated with arthroscopic-assisted mini-open rotator cuff repair. Eklem Hastalik Cerrahisi. 2011;22:134-9.

51. Holmgren T, Oberg B, Sjöberg I, Johansson K. Supervised strengthening exercises versus home-based movement exercises after arthroscopic acromioplasty: a randomized clinical trial. J Rehabil Med. 2012;44:12-8. https://doi.org/10.2340/16501977-0889.

52. Abdulla SY, Southerst D, Côté P, Shearer HM, Sutton D, Randhawa K, et al. Is exercise effective for the management of subacromial impingement syndrome and other soft tissue injuries of the shoulder? A systematic review by the Ontario protocol for traffic injury management (OPTIMa) collaboration. Man Ther. 2015;20:646-56. https://doi.org/10.1016/j.math. 2015.03.013.

53. Ingwersen KG, Jensen SL, Sørensen L, Jørgensen HR, Christensen R, Søgaard K, et al. Three months of progressive high-load versus traditional low-load strength training among patients with rotator cuff tendinopathy: primary results from the double-blind randomized controlled RoCTEx trial. Orthop J Sports Med. 2017;28(5):2325967117723292. https://doi. org/10.1177/2325967117723292. 
54. Maenhout AG, Mahieu NN, De Muynck M, De Wilde LF, Cools AM. Does adding heavy load eccentric training to rehabilitation of patients with unilateral subacromial impingement result in better outcome? A randomized, clinical trial. Knee Surg Sports Traumatol Arthrosc. 2013;21:115867. https://doi.org/10.1007/s00167-012-2012-8.

55. Hegedus EJ, Cook C, Lewis J, Wright A, Park JY. Combining orthopedic special tests to improve diagnosis of shoulder pathology. Phys Ther Sport. 2015;16:87-92. https://doi.org/10.1016/j.ptsp.2014.08.001.

56. Hegedus EJ, Goode A, Campbell S, Morin A, Tamaddoni M, Moorman CT 3rd, et al. Physical examination tests of the shoulder: a systematic review with meta-analysis of individual tests. Br J Sports Med. 2008;42:80-92; discussion 92. https://doi.org/10.1136/bjsm.2007.038406.

57. Vind M, Bogh SB, Larsen CM, Knudsen HK, Søgaard K, Juul-Kristensen B. Inter-examiner reproducibility of clinical tests and criteria used to identify subacromial impingement syndrome. BMJ Open. 2011;18(1):e000042. https://doi.org/10.1136/bmjopen-2010-000042.

58. Zheng F, Wang H, Gong H, Fan H, Zhang K, Du L. Role of ultrasound in the detection of rotator-cuff syndrome: an observational study. Med Sci Monit. 2019;6(25):5856-63. https://doi.org/10.12659/MSM.915547.

59. Lilje SC, Olander E, Berglund J, Skillgate E, Anderberg P. Experiences of older adults with Mobile phone text messaging as reminders of home exercises after specialized manual therapy for recurrent low Back pain: a qualitative study. JMIR Mhealth Uhealth. 2017;30(5):e39. https://doi.org/ 10.2196/mhealth.7184.

60. Andersen LL. Influence of psychosocial work environment on adherence to workplace exercise. J Occup Environ Med. 2011;53:182-4. https://doi. org/10.1097/JOM.0b013e3181207a01f.

61. Charbonnier C, Lädermann A, Kevelham B, Chagué S, Hoffmeyer P, Holzer N. Shoulder strengthening exercises adapted to specific shoulder pathologies can be selected using new simulation techniques: a pilot study. Int J Comput Assist Radiol Surg. 2018;13:321-30. https://doi.org/10, 1007/s11548-017-1668-4.

\section{Publisher's Note}

Springer Nature remains neutral with regard to jurisdictional claims in published maps and institutional affiliations.

- fast, convenient online submission

- thorough peer review by experienced researchers in your field

- rapid publication on acceptance

- support for research data, including large and complex data types

- gold Open Access which fosters wider collaboration and increased citations

- maximum visibility for your research: over $100 \mathrm{M}$ website views per year

At BMC, research is always in progress.

Learn more biomedcentral.com/submissions 